Kragujevac Journal of Mathematics

Volume 41(1) (2017), Pages 57-69.

\title{
NORMALIZED LAPLACIAN SPECTRUM OF DIFFERENT TYPE OF CORONAS OF TWO REGULAR GRAPHS
}

\author{
A. DAS ${ }^{1}$ AND P. PANIGRAHI ${ }^{1}$
}

\begin{abstract}
In this paper, we determine the full normalized Laplacian spectrum of the corona, edge corona and neighborhood corona of a connected regular graph with an arbitrary regular graph in terms of the normalized Laplacian eigenvalues of the original graphs. Moreover, applying these results we find some non-regular normalized Laplacian co-spectral graphs.
\end{abstract}

\section{INTRODUCTION}

There are several kinds of spectrums associated with a graph, for example, adjacency spectrum, Laplacian spectrum, signless Laplacian spectrum, normalized Laplacian spectrum etc. Normalized Laplacian spectrum determines the bipartiteness from the largest eigenvalue and the number of connected components from the second smallest eigenvalue [5]. F Chung [5] introduced the normalized Laplacian matrix of a graph $G$, denoted by $\mathcal{L}(G)$, which is a square matrix with rows and columns are indexed by vertices of $G$, and for any two vertices $u$ and $v$ of $G$ the $(u, v)^{t h}$ entry of it is given by

$$
\mathcal{L}(u, v)= \begin{cases}1, & \text { if } u=v \text { and } d_{v} \neq 0, \\ \frac{-1}{\sqrt{d_{u} d_{v}}}, & \text { if } u \text { and } v \text { are adjacent }, \\ 0, & \text { otherwise. }\end{cases}
$$

where $d_{u}$ and $d_{v}$ are degree of $u$ and $v$ respectively. If $D(G)$ is the diagonal matrix of vertex degrees and $A(G)$ is the adjacency matrix (that is $A(u, v)=1$ if and only if vertex $u$ is adjacent to vertex $v$ and 0 otherwise) of $G$, then we can write $\mathcal{L}(G)=I-D(G)^{-1 / 2} A(G) D(G)^{-1 / 2}$ with the convention that $D(G)^{-1}(u, u)=0$ if

Key words and phrases. Normalized Laplacian matrix, corona, edge corona, neighborhood corona, Kronecker product, Hadamard product.

2010 Mathematics Subject Classification. Primary: 05C50. Secondary: 47A75.

Received: February 3, 2016.

Accepted: August 5, 2016. 
$d_{u}=0$. We denote the characteristic polynomial $\operatorname{det}(\lambda I-\mathcal{L})$ of $\mathcal{L}(G)$ by $f_{G}(\lambda)$. The roots of $f_{G}(\lambda)$ are known as the normalized Laplacian eigenvalues of $G$. The multiset of the normalized Laplacian eigenvalues of $G$ is called the normalized Laplacian spectrum of $G$. Since $\mathcal{L}(G)$ is a symmetric and positive semi-definite matrix, its eigenvalues, denoted by $\lambda_{1}(G), \lambda_{2}(G), \ldots, \lambda_{n}(G)$, are all real, non-negative and can be arranged in non-decreasing order $\lambda_{1}(G) \leq \lambda_{2}(G) \leq \cdots \leq \lambda_{n}(G)$. Two graphs $G$ and $H$ are called cospectral if $A(G)$ and $A(H)$ have the same spectrum. Analogously graphs $G$ and $H$ are called normalized Laplacian cospectral or simply $\mathcal{L}$-cospectral if the spectrum of $\mathcal{L}(G)$ and $\mathcal{L}(H)$ are the same. In [5] Chung proved that all normalized Laplacian eigenvalues lie within the interval $[0,2]$ and 0 is always a normalized Laplacian eigenvalue of any graph $G$. She also determined normalized Laplacian spectrum of different kind of graphs like complete graphs, bipartite graphs, hypercubes etc. In [1], Banerjee and Jost investigated how the normalized Laplacian spectrum is affected by operations like motif doubling, graph splitting or joining. In [3], Butler and Grout produced (exponentially) large families of non-bipartite, non-regular graphs which are mutually cospectral, and also gave an example of a graph which is cospectral with its complement but is not self-complementary. In [11], Li studied the effect on the second smallest normalized Laplacian eigenvalue by grafting some pendant paths. In this paper we are interested on finding normalized Laplacian spectrum of some coronas of graphs, which are defined below.

Definition 1.1. For $i=1,2$, let $G_{i}$ be the graph with $n_{i}$ vertices and $m_{i}$ edges. Then (i) The corona [8] of $G_{1}$ and $G_{2}$, denoted by $G_{1} \circ G_{2}$, is the graph obtained by taking one copy of $G_{1}$ and $n_{1}$ copies of $G_{2}$, and then joining the $i^{t h}$ vertex of $G_{1}$ to every vertex in the $i^{\text {th }}$ copy of $G_{2}$ for $i=1,2, \ldots, n_{1}$. The corona $G_{1} \circ G_{2}$ has $n_{1}\left(n_{2}+1\right)$ vertices and $m_{1}+n_{1}\left(m_{2}+n_{2}\right)$ edges.

(ii) The edge corona [10] of $G_{1}$ and $G_{2}$, denoted by $G_{1} \diamond G_{2}$, is the graph obtained by taking one copy of $G_{1}$ and $m_{1}$ copies of $G_{2}$, and then joining two end vertices of the $i^{t h}$ edge of $G_{1}$ to every vertex in the $i^{t h}$ copy of $G_{2}$ for $i=1,2, \ldots, m_{1}$. The edge corona $G_{1} \diamond G_{2}$ has $n_{1}+m_{1} n_{2}$ vertices and $m_{1}+2 m_{1} n_{2}+m_{1} m_{2}$ edges.

(iii) The neighborhood corona [7] of $G_{1}$ and $G_{2}$, denoted by $G_{1} \star G_{2}$, is the graph obtained by taking one copy of $G_{1}$ and $n_{1}$ copies of $G_{2}$, and then joining every neighbor of the $i^{t h}$ vertex of $G_{1}$ to every vertex in the $i^{t h}$ copy of $G_{2}$ for $i=1,2, \ldots, n_{1}$. The neighborhood corona $G_{1} \star G_{2}$ has $n_{1}\left(n_{2}+1\right)$ vertices and $m_{1}\left(2 n_{2}+1\right)+n_{1} m_{2}$ edges.

Many researchers have worked on the corona, edge corona and neighborhood corona of two graphs. Barik et al. [2] provided complete information about the adjacency spectrum of $G_{1} \circ G_{2}$ for a connected graph $G_{1}$ and a regular graph $G_{2}$ and the Laplacian spectrum of $G_{1} \circ G_{2}$ for arbitrary graphs $G_{1}$ and $G_{2}$. Hou and Shiu [10] found the adjacency spectrum of $G_{1} \diamond G_{2}$ for a connected regular graph $G_{1}$ and a regular graph $G_{2}$ and the Laplacian spectrum of the same for a connected regular graph $G_{1}$ and a graph $G_{2}$. In [14], Wang and Zhou gave complete information about the signless Laplacian spectrum of $G_{1} \circ G_{2}$ for a graph $G_{1}$ and a regular graph $G_{2}$ and the signless 
Laplacian spectrum of $G_{1} \diamond G_{2}$ for a connected regular graph $G_{1}$ and a regular graph $G_{2}$. In [7], Gopalapillai determined the adjacency spectrum and Laplacian spectrum of $G_{1} \star G_{2}$ for a regular graph $G_{1}$ and an arbitrary graph $G_{2}$. Here we compute the full normalized Laplacian spectrum of $G_{1} \circ G_{2}, G_{1} \diamond G_{2}$ and $G_{1} \star G_{2}$ for two regular graphs $G_{1}$ and $G_{2}$, with $G_{1}$ connected.

To prove our results we need the following matrix products and few results on them. Recall that, the Kronecker product of matrices $A=\left(a_{i j}\right)$ of size $m \times n$ and $B$ of size $p \times q$, denoted by $A \otimes B$, is defined to be the $m p \times n q$ partition matrix $\left(a_{i j} B\right)$. It is known [9] that for matrices $M, N, P$ and $Q$ of suitable sizes, $M N \otimes P Q=(M \otimes P)(N \otimes Q)$. This implies that for nonsingular matrices $M$ and $N,(M \otimes N)^{-1}=M^{-1} \otimes N^{-1}$. It is also known that [9], for square matrices $M$ and $N$ of order $k$ and $s$ respectively, $\operatorname{det}(M \otimes N)=(\operatorname{det} M)^{s}(\operatorname{det} N)^{k}$. For two matrices $A$ and $B$, of same size $m \times n$, the Hadamard product $A \bullet B$ of $\mathrm{A}$ and $\mathrm{B}$ is a matrix of the same size $m \times n$ with entries given by $(A \bullet B)_{i j}=(A)_{i j} \cdot(B)_{i j}$ (entrywise multiplication). Hadamard product is commutative, that is $A \bullet B=B \bullet A$.

We also need the lemma below in the proof of our results.

Lemma 1.1 (Schur Complement [6]). If $Q$ is a non-singular square matrix and the order of all four matrices $M, N, P$ and $Q$ satisfy the rules of operations on matrices, then we have,

$$
\left|\begin{array}{ll}
M & N \\
P & Q
\end{array}\right|=\left|Q \| M-N Q^{-1} P\right|
$$

\section{Our Results}

Throughout the paper for any integer $k, I_{k}$ denotes the identity matrix of size $k$ and $J_{k}$ denotes the column vector of size $k$, whose all entries are 1 . In the lemma below we represent the normalized Laplacian matrix of corona, edge corona and neighborhood corona of two regular graphs in terms of Kronecker product and Hadamard product of matrices.

Lemma 2.1. For $i=1,2$, let $G_{i}$ be $r_{i}$-regular graph with order $n_{i}$ and size $m_{i}$. Then we have the following:

(i) $\mathcal{L}\left(G_{1}\right)=I_{n_{1}}-\frac{1}{r_{1}} A\left(G_{1}\right), \mathcal{L}\left(G_{2}\right)=I_{n_{2}}-\frac{1}{r_{2}} A\left(G_{2}\right)$.

(ii) $\mathcal{L}\left(G_{1} \circ G_{2}\right)=\left(\begin{array}{cc}\mathcal{L}\left(G_{1}\right) \bullet B\left(G_{1}\right) & -C_{n_{2}}^{T} \otimes I_{n_{1}} \\ -\left(C_{n_{2}}^{T} \otimes I_{n_{1}}\right)^{T} & \left(\mathcal{L}\left(G_{2}\right) \bullet B\left(G_{2}\right)\right) \otimes I_{n_{1}}\end{array}\right)$,

where $C_{n_{2}}$ is the column vector of size $n_{2}$ with all entries equal to $\frac{1}{\sqrt{\left(r_{1}+n_{2}\right)\left(r_{2}+1\right)}}, B\left(G_{1}\right)$ is the $n_{1} \times n_{1}$ matrix whose all diagonal entries are 1 and off-diagonal entries are $\frac{r_{1}}{r_{1}+n_{2}}$ and $B\left(G_{2}\right)$ is the $n_{2} \times n_{2}$ matrix whose all diagonal entries are 1 and off-diagonal entries are $\frac{r_{2}}{r_{2}+1}$.

(iii) $\mathcal{L}\left(G_{1} \diamond G_{2}\right)=\left(\begin{array}{cc}\mathcal{L}\left(G_{1}\right) \bullet B\left(G_{1}\right) & -R\left(G_{1}\right) \otimes C_{n_{2}}^{T} \\ -\left(R\left(G_{1}\right) \otimes C_{n_{2}}^{T}\right)^{T} & I_{m_{1}} \otimes\left(\mathcal{L}\left(G_{2}\right) \bullet B\left(G_{2}\right)\right)\end{array}\right)$,

where $C_{n_{2}}$ is the column vector of size $n_{2}$ with all entries equal to $\frac{1}{\sqrt{\left(r_{1}+r_{1} n_{2}\right)\left(r_{2}+2\right)}}$, 
$B\left(G_{1}\right)$ is the $n_{1} \times n_{1}$ matrix whose all diagonal entries are 1 and off-diagonal entries are $\frac{r_{1}}{r_{1}+r_{1} n_{2}}, B\left(G_{2}\right)$ is the $n_{2} \times n_{2}$ matrix whose all diagonal entries are 1 and offdiagonal entries are $\frac{r_{2}}{r_{2}+2}$ and $R\left(G_{1}\right)$ is the vertex-edge incidence matrix of $G_{1}$ of order $n_{1} \times m_{1}$ with entry $r_{i j}=1$ if vertex $i$ incident to the edge $j$ and 0 otherwise.

(iv) $\mathcal{L}\left(G_{1} \star G_{2}\right)=\left(\begin{array}{cc}\mathcal{L}\left(G_{1}\right) \bullet B\left(G_{1}\right) & -C_{n_{2}}^{T} \otimes A\left(G_{1}\right) \\ -\left(C_{n_{2}}^{T} \otimes A\left(G_{1}\right)\right)^{T} & \left(\mathcal{L}\left(G_{2}\right) \bullet B\left(G_{2}\right)\right) \otimes I_{n_{1}}\end{array}\right)$,

where $C_{n_{2}}$ is the column vector of size $n_{2}$ with all entries equal to $\frac{1}{\sqrt{\left(r_{1}+r_{1} n_{2}\right)\left(r_{2}+r_{1}\right)}}$, $B\left(G_{1}\right)$ is the $n_{1} \times n_{1}$ matrix whose all diagonal entries are 1 and off-diagonal entries are $\frac{r_{1}}{r_{1}+r_{1} n_{2}}$ and $B\left(G_{2}\right)$ is the $n_{2} \times n_{2}$ matrix whose all diagonal entries are 1 and off-diagonal entries are $\frac{r_{2}}{r_{2}+r_{1}}$.

Proof. Since $G_{1}$ and $G_{2}$ are $r_{1}$ and $r_{2}$ regular graphs respectively (i) is immediate.

(ii) For any two graphs $G_{1}$ and $G_{2}$, the adjacency matrix of $G_{1} \circ G_{2}$ is given by [2]:

$$
A\left(G_{1} \circ G_{2}\right)=\left(\begin{array}{cc}
A\left(G_{1}\right) & J_{n_{2}}^{T} \otimes I_{n_{1}} \\
\left(J_{n_{2}}^{T} \otimes I_{n_{1}}\right)^{T} & A\left(G_{2}\right) \otimes I_{n_{1}}
\end{array}\right) .
$$

Since $G_{1}$ is a $r_{1}$ regular graph with $n_{1}$ vertices and $G_{2}$ is a $r_{2}$ regular graph with $n_{2}$ vertices, the normalized Laplacian matrix of $G_{1} \circ G_{2}$ is

$$
\begin{aligned}
\mathcal{L}\left(G_{1} \circ G_{2}\right)= & I_{n_{1}\left(n_{2}+1\right)}-\left(\begin{array}{cc}
\frac{1}{\sqrt{r_{1}+n_{2}}} I_{n_{1}} & O \\
O & \frac{1}{\sqrt{r_{2}+1}} I_{n_{2}} \otimes I_{n_{1}}
\end{array}\right) \\
& \times\left(\begin{array}{cc}
A\left(G_{1}\right) & J_{n_{2}}^{T} \otimes I_{n_{1}} \\
\left(J_{n_{2}}^{T} \otimes I_{n_{1}}\right)^{T} & A\left(G_{2}\right) \otimes I_{n_{1}}
\end{array}\right) \times\left(\begin{array}{cc}
\frac{1}{\sqrt{r_{1}+n_{2}}} I_{n_{1}} & O \\
O & \frac{1}{\sqrt{r_{2}+1}} I_{n_{2}} \otimes I_{n_{1}}
\end{array}\right) \\
= & \left(\begin{array}{cc}
I_{n_{1}}-\frac{1}{r_{1}+n_{2}} A\left(G_{1}\right) & -C_{n_{2}}^{T} \otimes I_{n_{1}} \\
-\left(C_{n_{2}}^{T} \otimes I_{n_{1}}\right)^{T} & {\left[I_{n_{2}}-\frac{1}{r_{2}+1} A\left(G_{2}\right)\right] \otimes I_{n_{1}}}
\end{array}\right) \\
= & \left(\begin{array}{cc}
\mathcal{L}\left(G_{1}\right) \bullet B\left(G_{1}\right) & -C_{n_{2}}^{T} \otimes I_{n_{1}} \\
-\left(C_{n_{2}}^{T} \otimes I_{n_{1}}\right)^{T} & \left(\mathcal{L}\left(G_{2}\right) \bullet B\left(G_{2}\right)\right) \otimes I_{n_{1}}
\end{array}\right) .
\end{aligned}
$$

(iii) For any two graphs $G_{1}$ and $G_{2}$, the adjacency matrix of $G_{1} \diamond G_{2}$ is given by [10]:

$$
A\left(G_{1} \diamond G_{2}\right)=\left(\begin{array}{cc}
A\left(G_{1}\right) & R\left(G_{1}\right) \otimes J_{n_{2}}^{T} \\
\left(R\left(G_{1}\right) \otimes J_{n_{2}}^{T}\right)^{T} & I_{m_{1}} \otimes A\left(G_{2}\right)
\end{array}\right) .
$$

Since $G_{1}$ is a $r_{1}$ regular graph with $n_{1}$ vertices, $m_{1}$ edges and $G_{2}$ is a $r_{2}$ regular graph with $n_{2}$ vertices, $m_{2}$ edges, the normalized Laplacian matrix of $G_{1} \diamond G_{2}$ is

$$
\begin{aligned}
\mathcal{L}\left(G_{1} \diamond G_{2}\right)= & I_{n_{1}+m_{1} n_{2}}-\left(\begin{array}{cc}
\frac{1}{\sqrt{r_{1}+r_{1} n_{2}}} I_{n_{1}} & O \\
O & I_{m_{1}} \otimes \frac{1}{\sqrt{r_{2}+2}} I_{n_{2}}
\end{array}\right) \\
& \times\left(\begin{array}{ccc}
A\left(G_{1}\right) & R\left(G_{1}\right) \otimes J_{n_{2}}^{T} \\
\left(R\left(G_{1}\right) \otimes J_{n_{2}}^{T}\right)^{T} & I_{m_{1}} \otimes A\left(G_{2}\right)
\end{array}\right) \times\left(\begin{array}{cc}
\frac{1}{\sqrt{r_{1}+r_{1} n_{2}}} I_{n_{1}} & O \\
O & I_{m_{1}} \otimes \frac{1}{\sqrt{r_{2}+2}} I_{n_{2}}
\end{array}\right) \\
= & \left(\begin{array}{cc}
I_{n_{1}}-\frac{1}{r_{1}+r_{1} n_{2}} A\left(G_{1}\right) & -R\left(G_{1}\right) \otimes C_{n_{2}}^{T} \\
-\left(R\left(G_{1}\right) \otimes C_{n_{2}}^{T}\right)^{T} & I_{m_{1}} \otimes\left[I_{n_{2}}-\frac{1}{r_{2}+2} A\left(G_{2}\right)\right]
\end{array}\right)
\end{aligned}
$$




$$
=\left(\begin{array}{cc}
\mathcal{L}\left(G_{1}\right) \bullet B\left(G_{1}\right) & -R\left(G_{1}\right) \otimes C_{n_{2}}^{T} \\
-\left(R\left(G_{1}\right) \otimes C_{n_{2}}^{T}\right)^{T} & I_{m_{1}} \otimes\left(\mathcal{L}\left(G_{2}\right) \bullet B\left(G_{2}\right)\right)
\end{array}\right) .
$$

(iv) For any two graphs $G_{1}$ and $G_{2}$, the adjacency matrix of $G_{1} \star G_{2}$ is given by [7]:

$$
A\left(G_{1} \star G_{2}\right)=\left(\begin{array}{cc}
A\left(G_{1}\right) & J_{n_{2}}^{T} \otimes A\left(G_{1}\right) \\
\left(J_{n_{2}}^{T} \otimes A\left(G_{1}\right)\right)^{T} & A\left(G_{2}\right) \otimes I_{n_{1}}
\end{array}\right) .
$$

Since $G_{1}$ is a $r_{1}$ regular graph with $n_{1}$ vertices and $G_{2}$ is a $r_{2}$ regular graph with $n_{2}$ vertices, the normalized Laplacian matrix of $G_{1} \star G_{2}$ will be

$$
\begin{aligned}
\mathcal{L}\left(G_{1} \star G_{2}\right)= & I_{n_{1}\left(n_{2}+1\right)}-\left(\begin{array}{cc}
\frac{1}{\sqrt{r_{1}+r_{1} n_{2}}} I_{n_{1}} & O \\
O & \frac{1}{\sqrt{r_{2}+r_{1}}} I_{n_{2}} \otimes I_{n_{1}}
\end{array}\right) \\
& \times\left(\begin{array}{cc}
A\left(G_{1}\right) & J_{n_{2}}^{T} \otimes A\left(G_{1}\right) \\
\left(J_{n_{2}}^{T} \otimes A\left(G_{1}\right)\right)^{T} & A\left(G_{2}\right) \otimes I_{n_{1}}
\end{array}\right) \times\left(\begin{array}{cc}
\frac{1}{\sqrt{r_{1}+r_{1} n_{2}}} I_{n_{1}} & O \\
O & \frac{1}{\sqrt{r_{2}+r_{1}}} I_{n_{2}} \otimes I_{n_{1}}
\end{array}\right) \\
= & \left(\begin{array}{cc}
I_{n_{1}}-\frac{1}{r_{1}+r_{1} n_{2}} A\left(G_{1}\right) & -C_{n_{2}}^{T} \otimes A\left(G_{1}\right) \\
-\left(C_{n_{2}}^{T} \otimes A\left(G_{1}\right)\right)^{T} & {\left[I_{n_{2}}-\frac{1}{r_{2}+r_{1}} A\left(G_{2}\right)\right] \otimes I_{n_{1}}}
\end{array}\right) \\
= & \left(\begin{array}{cc}
\mathcal{L}\left(G_{1}\right) \bullet B\left(G_{1}\right) & -C_{n_{2}}^{T} \otimes A\left(G_{1}\right) \\
-\left(C_{n_{2}}^{T} \otimes A\left(G_{1}\right)\right)^{T} & \left(\mathcal{L}\left(G_{2}\right) \bullet B\left(G_{2}\right)\right) \otimes I_{n_{1}}
\end{array}\right) .
\end{aligned}
$$

Notation 2.1. Let $G$ be a graph on $n$ vertices, $B$ and $C$ be matrices of size $n \times n$ and $n \times 1$ respectively. For any parameter $\lambda$, we have the notation

$$
\chi_{G}(B, C, \lambda)=C^{T}\left(\lambda I_{n}-(\mathcal{L}(G) \bullet B)\right)^{-1} C .
$$

We note that the notation is similar to the notion 'coronal' which was introduced by McLeman[13].

Theorem 2.1. For $i=1,2$, let $G_{i}$ be $r_{i}$-regular graph on $n_{i}$ vertices with $G_{1}$ connected. Then the normalized Laplacian spectrum of $G_{1} \circ G_{2}$ consists of

(i) the eigenvalue $\frac{1+r_{2} \delta_{j}}{r_{2}+1}$ with multiplicity $n_{1}$ for every eigenvalue $\delta_{j}, j=2, \ldots, n_{2}$, of $\mathcal{L}\left(G_{2}\right)$;

(ii) two simple eigenvalues

$$
\frac{\left(2 n_{2}+n_{2} r_{2}+r_{1}+r_{1} \mu_{i}+r_{1} r_{2} \mu_{i}\right) \pm \sqrt{\left(2 n_{2}+n_{2} r_{2}+r_{1}+r_{1} \mu_{i}+r_{1} r_{2} \mu_{i}\right)^{2}-4 r_{1} \mu_{i}\left(r_{2}+1\right)\left(r_{1}+n_{2}\right)}}{2\left(r_{2}+1\right)\left(r_{1}+n_{2}\right)},
$$

for each eigenvalue $\mu_{i}, i=1,2, \ldots, n_{1}$, of $\mathcal{L}\left(G_{1}\right)$.

Proof. The normalized Laplacian characteristic polynomial of $G_{1} \circ G_{2}$ is

$$
\begin{aligned}
& f_{G_{1} \circ G_{2}}(\lambda) \\
= & \operatorname{det}\left(\lambda I_{n_{1}\left(n_{2}+1\right)}-\mathcal{L}\left(G_{1} \circ G_{2}\right)\right) \\
= & \operatorname{det}\left(\begin{array}{cc}
\lambda I_{n_{1}}-\left(\mathcal{L}\left(G_{1}\right) \bullet B\left(G_{1}\right)\right) & C_{n_{2}}^{T} \otimes I_{n_{1}} \\
\left(C_{n_{2}}^{T} \otimes I_{n_{1}}\right)^{T} & \lambda I_{n_{1} n_{2}}-\left(\left(\mathcal{L}\left(G_{2}\right) \bullet B\left(G_{2}\right)\right) \otimes I_{n_{1}}\right)
\end{array}\right) \\
= & \operatorname{det}\left(\begin{array}{cc}
\lambda I_{n_{1}}-\left(\mathcal{L}\left(G_{1}\right) \bullet B\left(G_{1}\right)\right) & C_{n_{2}}^{T} \otimes I_{n_{1}} \\
\left(C_{n_{2}}^{T} \otimes I_{n_{1}}\right)^{T} & \left(\lambda I_{n_{2}}-\left(\mathcal{L}\left(G_{2}\right) \bullet B\left(G_{2}\right)\right)\right) \otimes I_{n_{1}}
\end{array}\right)
\end{aligned}
$$




$$
\begin{aligned}
& =\operatorname{det}\left(\left(\lambda I_{n_{2}}-\left(\mathcal{L}\left(G_{2}\right) \bullet B\left(G_{2}\right)\right)\right) \otimes I_{n_{1}}\right) \\
& \times \operatorname{det}\left(\lambda I_{n_{1}}-\left(\mathcal{L}\left(G_{1}\right) \bullet B\left(G_{1}\right)\right)-\left(C_{n_{2}}^{T} \otimes I_{n_{1}}\right)\right. \\
& \left.\times\left(\left(\lambda I_{n_{2}}-\left(\mathcal{L}\left(G_{2}\right) \bullet B\left(G_{2}\right)\right)\right) \otimes I_{n_{1}}\right)^{-1}\left(C_{n_{2}}^{T} \otimes I_{n_{1}}\right)^{T}\right) \quad \text { [by Lemma 1.1] } \\
& =\operatorname{det}\left(\lambda I_{n_{2}}-\left(\mathcal{L}\left(G_{2}\right) \bullet B\left(G_{2}\right)\right)\right)^{n_{1}} \\
& \times \operatorname{det}\left(\lambda I_{n_{1}}-\left(\mathcal{L}\left(G_{1}\right) \bullet B\left(G_{1}\right)\right)-\left(C_{n_{2}}^{T}\left(\lambda I_{n_{2}}-\left(\mathcal{L}\left(G_{2}\right) \bullet B\left(G_{2}\right)\right)\right)^{-1} C_{n_{2}}\right) \otimes I_{n_{1}}\right) \\
& =\operatorname{det}\left(\lambda I_{n_{2}}-\left(\mathcal{L}\left(G_{2}\right) \bullet B\left(G_{2}\right)\right)\right)^{n_{1}} \\
& \times \operatorname{det}\left(\left(\lambda-\chi_{G_{2}}\left(B\left(G_{2}\right), C_{n_{2}}, \lambda\right)\right) I_{n_{1}}-\left(\mathcal{L}\left(G_{1}\right) \bullet B\left(G_{1}\right)\right)\right) \quad \text { [from Notation 2.1]. }
\end{aligned}
$$

Since $\mathcal{L}\left(G_{2}\right) \bullet B\left(G_{2}\right)=I_{n_{2}}-\frac{1}{r_{2}+1} A\left(G_{2}\right)$, one gets $\mathcal{L}\left(G_{2}\right) \bullet B\left(G_{2}\right)=\frac{1}{r_{2}+1}\left(I_{n_{2}}+r_{2} \mathcal{L}\left(G_{2}\right)\right)$. As $G_{2}$ is regular, the sum of all entries on every row of $\mathcal{L}\left(G_{2}\right)$ is zero. That means $\mathcal{L}\left(G_{2}\right) C_{n_{2}}=\left(1-\frac{r_{2}}{r_{2}}\right) C_{n_{2}}=0 C_{n_{2}}$. Then

$$
\left(\mathcal{L}\left(G_{2}\right) \bullet B\left(G_{2}\right)\right) C_{n_{2}}=\left(1-\frac{r_{2}}{r_{2}+1}\right) C_{n_{2}}=\frac{1}{r_{2}+1} C_{n_{2}}
$$

and

$$
\left(\lambda I_{n_{2}}-\left(\mathcal{L}\left(G_{2}\right) \bullet B\left(G_{2}\right)\right)\right) C_{n_{2}}=\left(\lambda-\frac{1}{r_{2}+1}\right) C_{n_{2}} .
$$

Also, $C_{n_{2}}^{T} C_{n_{2}}=\frac{n_{2}}{\left(r_{1}+n_{2}\right)\left(r_{2}+1\right)}$. Therefore,

$$
\begin{aligned}
\chi_{G_{2}}\left(B\left(G_{2}\right), C_{n_{2}}, \lambda\right) & =C_{n_{2}}^{T}\left(\lambda I_{n_{2}}-\left(\mathcal{L}\left(G_{2}\right) \bullet B\left(G_{2}\right)\right)\right)^{-1} C_{n_{2}} \\
& =\frac{C_{n_{2}}^{T} C_{n_{2}}}{\left(\lambda-\frac{1}{r_{2}+1}\right)}=\frac{n_{2}}{\left(r_{1}+n_{2}\right)\left(r_{2}+1\right)\left(\lambda-\frac{1}{r_{2}+1}\right)} .
\end{aligned}
$$

Again, since $\mathcal{L}\left(G_{1}\right) \bullet B\left(G_{1}\right)=I_{n_{1}}-\frac{1}{r_{1}+n_{2}} A\left(G_{1}\right)$, then $\mathcal{L}\left(G_{1}\right) \bullet B\left(G_{1}\right)=\frac{1}{r_{1}+n_{2}}\left(n_{2} I_{n_{2}}+\right.$ $\left.r_{1} \mathcal{L}\left(G_{1}\right)\right)$.

Now, if $\delta_{j}$ is an eigenvalue of $\mathcal{L}\left(G_{2}\right)$ and $\mu_{i}$ is an eigenvalue of $\mathcal{L}\left(G_{1}\right)$ then

$$
\begin{aligned}
f_{G_{1} \circ G_{2}}(\lambda)= & \prod_{j=1}^{n_{2}}\left(\lambda-\frac{1+r_{2} \delta_{j}}{r_{2}+1}\right)^{n_{1}} \\
& \times \prod_{i=1}^{n_{1}}\left(\lambda-\frac{n_{2}}{\left(r_{1}+n_{2}\right)\left(r_{2}+1\right)\left(\lambda-\frac{1}{r_{2}+1}\right)}-\frac{n_{2}+r_{1} \mu_{i}}{r_{1}+n_{2}}\right) .
\end{aligned}
$$

(i) Since the only pole of $\chi_{G_{2}}\left(B\left(G_{2}\right), C_{n_{2}}, \lambda\right)=\frac{n_{2}}{\left(r_{1}+n_{2}\right)\left(r_{2}+1\right)\left(\lambda-\frac{1}{r_{2}+1}\right)}$ is $\lambda=\frac{1}{r_{2}+1}$ and 0 is an eigenvalue of $\mathcal{L}\left(G_{2}\right)$, then $\frac{1+r_{2} \delta_{j}}{r_{2}+1}$ is an eigenvalue of $\mathcal{L}\left(G_{1} \circ G_{2}\right)$ with multiplicity $n_{1}$ for $j=2, \ldots, n_{2}$.

(ii) The remaining $2 n_{1}$ eigenvalues are obtained by solving the equation

$$
\lambda-\frac{n_{2}}{\left(r_{1}+n_{2}\right)\left(r_{2}+1\right)\left(\lambda-\frac{1}{r_{2}+1}\right)}-\frac{n_{2}+r_{1} \mu_{i}}{r_{1}+n_{2}}=0
$$


or

$$
\left(r_{2}+1\right)\left(r_{1}+n_{2}\right) \lambda^{2}-\left(2 n_{2}+n_{2} r_{2}+r_{1}+r_{1} \mu_{i}+r_{1} r_{2} \mu_{i}\right) \lambda+r_{1} \mu_{i}=0 .
$$

So the eigenvalues are,

$$
\lambda_{i}=\frac{\left(2 n_{2}+n_{2} r_{2}+r_{1}+r_{1} \mu_{i}+r_{1} r_{2} \mu_{i}\right) \pm \sqrt{\left(2 n_{2}+n_{2} r_{2}+r_{1}+r_{1} \mu_{i}+r_{1} r_{2} \mu_{i}\right)^{2}-4 r_{1} \mu_{i}\left(r_{2}+1\right)\left(r_{1}+n_{2}\right)}}{2\left(r_{2}+1\right)\left(r_{1}+n_{2}\right)},
$$

for $i=1,2, \ldots, n_{1}$.

Theorem 2.2. For $i=1,2$, let $G_{i}$ be $r_{i}$-regular graph on $n_{i}$ vertices and $m_{i}$ edges with $G_{1}$ connected. Then the normalized Laplacian spectrum of $G_{1} \diamond G_{2}$ consists of (i) the eigenvalue $\frac{2+r_{2} \delta_{j}}{r_{2}+2}$ with multiplicity $m_{1}$ for every eigenvalue $\delta_{j}, j=2, \ldots, n_{2}$, of $\mathcal{L}\left(G_{2}\right)$;

(ii) two simple eigenvalues

$$
\frac{\left(2+4 n_{2}+r_{2} n_{2}+r_{2} \mu_{i}+2 \mu_{i}\right) \pm \sqrt{\left(2+4 n_{2}+r_{2} n_{2}+r_{2} \mu_{i}+2 \mu_{i}\right)^{2}-4 \mu_{i}\left(n_{2}+2\right)\left(r_{2}+2\right)\left(n_{2}+1\right)}}{2\left(r_{2}+2\right)\left(n_{2}+1\right)},
$$

for each eigenvalue $\mu_{i}, i=1,2, \ldots, n_{1}$, of $\mathcal{L}\left(G_{1}\right)$;

(iii) the eigenvalue $\frac{2}{r_{2}+2}$ with multiplicity $\left(m_{1}-n_{1}\right)$ if $n_{1}<m_{1}$.

Proof. The normalized Laplacian characteristic polynomial of $G_{1} \diamond G_{2}$ is

$$
f_{G_{1} \diamond G_{2}}(\lambda)
$$$$
=\operatorname{det}\left(\lambda I_{n_{1}+m_{1} n_{2}}-\mathcal{L}\left(G_{1} \diamond G_{2}\right)\right)
$$$$
=\operatorname{det}\left(\begin{array}{cc}
\lambda I_{n_{1}}-\left(\mathcal{L}\left(G_{1}\right) \bullet B\left(G_{1}\right)\right) & R\left(G_{1}\right) \otimes C_{n_{2}}^{T} \\
\left(R\left(G_{1}\right) \otimes C_{n_{2}}^{T}\right)^{T} & \lambda I_{m_{1} n_{2}}-I_{m_{1}} \otimes\left(\left(\mathcal{L}\left(G_{2}\right) \bullet B\left(G_{2}\right)\right)\right)
\end{array}\right)
$$$$
=\operatorname{det}\left(\begin{array}{cc}
\lambda I_{n_{1}}-\left(\mathcal{L}\left(G_{1}\right) \bullet B\left(G_{1}\right)\right) & R\left(G_{1}\right) \otimes C_{n_{2}}^{T} \\
\left(R\left(G_{1}\right) \otimes C_{n_{2}}^{T}\right)^{T} & I_{m_{1}} \otimes\left(\lambda I_{n_{2}}-\left(\mathcal{L}\left(G_{2}\right) \bullet B\left(G_{2}\right)\right)\right)
\end{array}\right)
$$

$$
\begin{aligned}
=\operatorname{det} & \left(\left(\lambda I_{n_{2}}-\left(\mathcal{L}\left(G_{2}\right) \bullet B\left(G_{2}\right)\right)\right) \otimes I_{m_{1}}\right) \operatorname{det}\left(\lambda I_{n_{1}}-\left(\mathcal{L}\left(G_{1}\right) \bullet B\left(G_{1}\right)\right)-\left(R\left(G_{1}\right) \otimes C_{n_{2}}^{T}\right)\right. \\
& \times\left(I_{m_{1}} \otimes\left(\lambda I_{n_{2}}-\left(\mathcal{L}\left(G_{2}\right) \bullet B\left(G_{2}\right)\right)\right)^{-1}\left(R\left(G_{1}\right) \otimes C_{n_{2}}^{T}\right)^{T}\right) \quad \text { [by Lemma 1.1] }
\end{aligned}
$$

$=\operatorname{det}\left(\lambda I_{n_{2}}-\left(\mathcal{L}\left(G_{2}\right) \bullet B\left(G_{2}\right)\right)\right)^{m_{1}} \times \operatorname{det}\left(\lambda I_{n_{1}}-\left(\mathcal{L}\left(G_{1}\right) \bullet B\left(G_{1}\right)\right)\right.$

$$
\left.-\left(R\left(G_{1}\right) \otimes C_{n_{2}}^{T}\right)\left(I_{m_{1}} \otimes\left(\lambda I_{n_{2}}-\left(\mathcal{L}\left(G_{2}\right) \bullet B\left(G_{2}\right)\right)\right)\right)^{-1}\left(R\left(G_{1}\right) \otimes C_{n_{2}}^{T}\right)^{T}\right)
$$

$$
\begin{aligned}
=\operatorname{det} & \left(\lambda I_{n_{2}}-\left(\mathcal{L}\left(G_{2}\right) \bullet B\left(G_{2}\right)\right)\right)^{m_{1}} \operatorname{det}\left(\lambda I_{n_{1}}-\left(\mathcal{L}\left(G_{1}\right) \bullet B\left(G_{1}\right)\right)\right. \\
& \left.-\left(R\left(G_{1}\right) I_{m_{1}} R\left(G_{1}\right)^{T}\right) \otimes\left(C_{n_{2}}^{T}\left(\lambda I_{n_{2}}-\left(\mathcal{L}\left(G_{2}\right) \bullet B\left(G_{2}\right)\right)\right)^{-1} C_{n_{2}}\right)\right)
\end{aligned}
$$

[It is well known [6] that $R\left(G_{1}\right) R\left(G_{1}\right)^{T}=A\left(G_{1}\right)+r_{1} I_{n_{1}}$ and $A\left(G_{1}\right)=$ $r_{1}\left(I_{n_{1}}-\mathcal{L}\left(G_{1}\right)\right)$, so one gets $\left.R\left(G_{1}\right) R\left(G_{1}\right)^{T}=r_{1}\left(2 I_{n_{1}}-\mathcal{L}\left(G_{1}\right)\right)\right]$

$$
\begin{aligned}
& =\operatorname{det}\left(\lambda I_{n_{2}}-\left(\mathcal{L}\left(G_{2}\right) \bullet B\left(G_{2}\right)\right)\right)^{m_{1}} \operatorname{det}\left(\lambda I_{n_{1}}-\left(\mathcal{L}\left(G_{1}\right) \bullet B\left(G_{1}\right)\right)\right. \\
& \left.\quad-r_{1}\left(2 I_{n_{1}}-\mathcal{L}\left(G_{1}\right)\right) \otimes \chi_{G_{2}}\left(B\left(G_{2}\right), C_{n_{2}}, \lambda\right)\right) \\
& =\operatorname{det}\left(\lambda I_{n_{2}}-\left(\mathcal{L}\left(G_{2}\right) \bullet B\left(G_{2}\right)\right)\right)^{m_{1}} \operatorname{det}\left(\left(\lambda-2 r_{1} \chi_{G_{2}}\left(B\left(G_{2}\right), C_{n_{2}}, \lambda\right)\right) I_{n_{1}}\right. \\
& \left.\quad-\left(\mathcal{L}\left(G_{1}\right) \bullet B\left(G_{1}\right)\right)+r_{1} \chi_{G_{2}}\left(B\left(G_{2}\right), C_{n_{2}}, \lambda\right) \mathcal{L}\left(G_{1}\right)\right) .
\end{aligned}
$$$$
\text { [from Notation 2.1] }
$$

Since $\mathcal{L}\left(G_{2}\right) \bullet B\left(G_{2}\right)=I_{n_{2}}-\frac{1}{r_{2}+2} A\left(G_{2}\right)$, we get $\mathcal{L}\left(G_{2}\right) \bullet B\left(G_{2}\right)=\frac{1}{r_{2}+2}\left(2 I_{n_{2}}+r_{2} \mathcal{L}\left(G_{2}\right)\right)$. The sum of all entries on every row of $\mathcal{L}\left(G_{2}\right)$ is zero because $G_{2}$ is regular. That 
means $\mathcal{L}\left(G_{2}\right) C_{n_{2}}=\left(1-\frac{r_{2}}{r_{2}}\right) C_{n_{2}}=0 C_{n_{2}}$. Then

$$
\left(\mathcal{L}\left(G_{2}\right) \bullet B\left(G_{2}\right)\right) C_{n_{2}}=\left(1-\frac{r_{2}}{r_{2}+2}\right) C_{n_{2}}=\frac{2}{r_{2}+2} C_{n_{2}}
$$

and

$$
\left(\lambda I_{n_{2}}-\left(\mathcal{L}\left(G_{2}\right) \bullet B\left(G_{2}\right)\right)\right) C_{n_{2}}=\left(\lambda-\frac{2}{r_{2}+2}\right) C_{n_{2}}
$$

Also, $C_{n_{2}}^{T} C_{n_{2}}=\frac{n_{2}}{\left(r_{1}+r_{1} n_{2}\right)\left(r_{2}+2\right)}$. Hence,

$$
\begin{aligned}
\chi_{G_{2}}\left(B\left(G_{2}\right), C_{n_{2}}, \lambda\right) & =C_{n_{2}}^{T}\left(\lambda I_{n_{2}}-\left(\mathcal{L}\left(G_{2}\right) \bullet B\left(G_{2}\right)\right)\right)^{-1} C_{n_{2}} \\
& =\frac{n_{2}}{\left(r_{1}+r_{1} n_{2}\right)\left(r_{2}+2\right)\left(\lambda-\frac{2}{r_{2}+2}\right)} .
\end{aligned}
$$

As $\mathcal{L}\left(G_{1}\right) \bullet B\left(G_{1}\right)=I_{n_{1}}-\frac{1}{r_{1}+r_{1} n_{2}} A\left(G_{1}\right)$, we have

$$
\mathcal{L}\left(G_{1}\right) \bullet B\left(G_{1}\right)=\frac{1}{r_{1}+r_{1} n_{2}}\left(r_{1} n_{2} I_{n_{2}}+r_{1} \mathcal{L}\left(G_{1}\right)\right)=\frac{1}{n_{2}+1}\left(n_{2} I_{n_{2}}+\mathcal{L}\left(G_{1}\right)\right) .
$$

Now, if $\delta_{j}$ is an eigenvalue of $\mathcal{L}\left(G_{2}\right)$ and $\mu_{i}$ is an eigenvalue of $\mathcal{L}\left(G_{1}\right)$ then,

$$
\begin{aligned}
f_{G_{1} \diamond G_{2}}(\lambda)= & \prod_{j=1}^{n_{2}}\left(\lambda-\frac{2+r_{2} \delta_{j}}{r_{2}+2}\right)^{m_{1}} \prod_{j=1}^{n_{1}}\left(\lambda-\frac{2 n_{2}}{\left(n_{2}+1\right)\left(r_{2}+2\right)\left(\lambda-\frac{2}{r_{2}+2}\right)}\right. \\
& \left.-\frac{n_{2}+\mu_{i}}{n_{2}+1}+\frac{n_{2} \mu_{i}}{\left(n_{2}+1\right)\left(r_{2}+2\right)\left(\lambda-\frac{2}{r_{2}+2}\right)}\right) .
\end{aligned}
$$

(i) Since the only pole of $\chi_{G_{2}}\left(B\left(G_{2}\right), C_{n_{2}}, \lambda\right)=\frac{n_{2}}{\left(r_{1}+r_{1} n_{2}\right)\left(r_{2}+2\right)\left(\lambda-\frac{2}{r_{2}+2}\right)}$ is $\lambda=\frac{2}{r_{2}+2}$ and 0 is an eigenvalue of $\mathcal{L}\left(G_{2}\right)$, one gets that $\frac{2+r_{2} \delta_{j}}{r_{2}+2}$ is an eigenvalue of $\mathcal{L}\left(G_{1} \diamond G_{2}\right)$ with multiplicity $m_{1}$ for $j=2, \ldots, n_{2}$.

(ii) The $2 n_{1}$ eigenvalues are obtained by solving the equation

$$
\lambda-\frac{2 n_{2}}{\left(n_{2}+1\right)\left(r_{2}+2\right)\left(\lambda-\frac{2}{r_{2}+2}\right)}-\frac{n_{2}+\mu_{i}}{n_{2}+1}+\frac{n_{2} \mu_{i}}{\left(n_{2}+1\right)\left(r_{2}+2\right)\left(\lambda-\frac{2}{r_{2}+2}\right)}=0 .
$$

or

$$
\left(n_{2}+1\right)\left(r_{2}+2\right) \lambda^{2}-\left(2+4 n_{2}+r_{2} n_{2}+r_{2} \mu_{i}+2 \mu_{i}\right) \lambda+\mu_{i}\left(n_{2}+2\right)=0 .
$$

So the eigenvalues are,

$$
\lambda_{i}=\frac{\left(2+4 n_{2}+r_{2} n_{2}+r_{2} \mu_{i}+2 \mu_{i}\right) \pm \sqrt{\left(2+4 n_{2}+r_{2} n_{2}+r_{2} \mu_{i}+2 \mu_{i}\right)^{2}-4 \mu_{i}\left(n_{2}+2\right)\left(r_{2}+2\right)\left(n_{2}+1\right)}}{2\left(r_{2}+2\right)\left(n_{2}+1\right)}
$$

for $i=1,2, \ldots, n_{1}$. 
(iii) Since $G_{1}$ is connected regular graph, then $n_{1} \leq m_{1}$. If $n_{1}=m_{1}$ then all eigenvalues are obtained by $(i)$ and $(i i)$. If $n_{1}<m_{1}$ then the remaining $n_{1}+m_{1} n_{2}-m_{1}\left(n_{2}-\right.$ 1) $-2 n_{1}=m_{1}-n_{1}$ normalized Laplacian eigenvalues of $G$ must come from the only pole $\lambda=\frac{2}{r_{2}+2}$ of $\chi_{G_{2}}\left(B\left(G_{2}\right), C_{n_{2}}, \lambda\right)=\frac{n_{2}}{\left(r_{1}+r_{1} n_{2}\right)\left(r_{2}+2\right)\left(\lambda-\frac{2}{r_{2}+2}\right)}$.

Theorem 2.3. For $i=1,2$, let $G_{i}$ be $r_{i}$-regular graph on $n_{i}$ vertices with $G_{1}$ connected. Then the normalized Laplacian spectrum of $G_{1} \star G_{2}$ consists of

(i) the eigenvalue $\frac{r_{1}+r_{2} \delta_{j}}{r_{2}+r_{1}}$ with multiplicity $n_{1}$ for every eigenvalue $\delta_{j}, j=2, \ldots, n_{2}$, of $\mathcal{L}\left(G_{2}\right)$;

(ii) two simple eigenvalues

$$
\frac{\left(r_{1}+2 r_{1} n_{2}+r_{2} n_{2}+r_{1} \mu_{i}+r_{2} \mu_{i}\right) \pm \sqrt{\left(r_{1}+2 r_{1} n_{2}+r_{2} n_{2}+r_{1} \mu_{i}+r_{2} \mu_{i}\right)^{2}-4 r_{1} \mu_{i}\left(r_{2}+r_{1}\right)\left(n_{2}+1\right)\left(1-n_{2} \mu_{i}+2 n_{2}\right)}}{2\left(r_{2}+r_{1}\right)\left(n_{2}+1\right)}
$$

for each eigenvalue $\mu_{i}, i=1,2, \ldots, n_{1}$, of $\mathcal{L}\left(G_{1}\right)$.

Proof. The normalized Laplacian characteristic polynomial of $G_{1} \star G_{2}$ is

$$
\begin{aligned}
& f_{G_{1} \star G_{2}}(\lambda)=\operatorname{det}\left(\lambda I_{n_{1}\left(n_{2}+1\right)}-\mathcal{L}\left(G_{1} \star G_{2}\right)\right) \\
& =\operatorname{det}\left(\begin{array}{cc}
\lambda I_{n_{1}}-\left(\mathcal{L}\left(G_{1}\right) \bullet B\left(G_{1}\right)\right) & C_{n_{2}}^{T} \otimes A\left(G_{1}\right) \\
\left(C_{n_{2}}^{T} \otimes A\left(G_{1}\right)\right)^{T} & \lambda I_{n_{1} n_{2}}-\left(\left(\mathcal{L}\left(G_{2}\right) \bullet B\left(G_{2}\right)\right) \otimes I_{n_{1}}\right)
\end{array}\right) \\
& =\operatorname{det}\left(\begin{array}{cc}
\lambda I_{n_{1}}-\left(\mathcal{L}\left(G_{1}\right) \bullet B\left(G_{1}\right)\right) & C_{n_{2}}^{T} \otimes A\left(G_{1}\right) \\
\left(C_{n_{2}}^{T} \otimes A\left(G_{1}\right)\right)^{T} & \left(\lambda I_{n_{2}}-\left(\mathcal{L}\left(G_{2}\right) \bullet B\left(G_{2}\right)\right)\right) \otimes I_{n_{1}}
\end{array}\right) \\
& =\operatorname{det}\left(\left(\lambda I_{n_{2}}-\left(\mathcal{L}\left(G_{2}\right) \bullet B\left(G_{2}\right)\right)\right) \otimes I_{n_{1}}\right) \\
& \times \operatorname{det}\left(\lambda I_{n_{1}}-\left(\mathcal{L}\left(G_{1}\right) \bullet B\left(G_{1}\right)\right)-\left(C_{n_{2}}^{T} \otimes A\left(G_{1}\right)\right)\right. \\
& \left.\times\left(\left(\lambda I_{n_{2}}-\left(\mathcal{L}\left(G_{2}\right) \bullet B\left(G_{2}\right)\right)\right) \otimes I_{n_{1}}\right)^{-1}\left(C_{n_{2}}^{T} \otimes A\left(G_{1}\right)\right)^{T}\right) \quad \text { [by Lemma 1.1] } \\
& =\operatorname{det}\left(\lambda I_{n_{2}}-\left(\mathcal{L}\left(G_{2}\right) \bullet B\left(G_{2}\right)\right)\right)^{n_{1}} \operatorname{det}\left(\lambda I_{n_{1}}-\left(\mathcal{L}\left(G_{1}\right) \bullet B\left(G_{1}\right)\right)\right. \\
& \left.-\left(C_{n_{2}}^{T}\left(\lambda I_{n_{2}}-\left(\mathcal{L}\left(G_{2}\right) \bullet B\left(G_{2}\right)\right)\right)^{-1} C_{n_{2}}\right) \otimes A\left(G_{1}\right)^{2}\right) \\
& =\operatorname{det}\left(\lambda I_{n_{2}}-\left(\mathcal{L}\left(G_{2}\right) \bullet B\left(G_{2}\right)\right)\right)^{n_{1}} \operatorname{det}\left(\lambda I_{n_{1}}\right. \\
& \text { - } \left.\left(\mathcal{L}\left(G_{1}\right) \bullet B\left(G_{1}\right)\right)-\chi_{G_{2}}\left(B\left(G_{2}\right), C_{n_{2}}, \lambda\right) A\left(G_{1}\right)^{2}\right) \quad \text { [from Notation 2.1] } \\
& =\operatorname{det}\left(\lambda I_{n_{2}}-\left(\mathcal{L}\left(G_{2}\right) \bullet B\left(G_{2}\right)\right)\right)^{n_{1}} \operatorname{det}\left(\lambda I_{n_{1}}-\left(\mathcal{L}\left(G_{1}\right) \bullet B\left(G_{1}\right)\right)\right. \\
& \text { - } \left.\chi_{G_{2}}\left(B\left(G_{2}\right), C_{n_{2}}, \lambda\right) r_{1}^{2}\left(I_{n_{1}}-\mathcal{L}\left(G_{1}\right)\right)^{2}\right) \quad\left[\text { as } A\left(G_{1}\right)=r_{1}\left(I_{n_{1}}-\mathcal{L}\left(G_{1}\right)\right)\right] \text {. }
\end{aligned}
$$

Since $\mathcal{L}\left(G_{2}\right) \bullet B\left(G_{2}\right)=I_{n_{2}}-\frac{1}{r_{2}+r_{1}} A\left(G_{2}\right)$, we have $\mathcal{L}\left(G_{2}\right) \bullet B\left(G_{2}\right)=\frac{1}{r_{2}+r_{1}}\left(r_{1} I_{n_{2}}+\right.$ $\left.r_{2} \mathcal{L}\left(G_{2}\right)\right)$. As $G_{2}$ is regular, the sum of all entries on every row of $\mathcal{L}\left(G_{2}\right)$ is zero. That means, $\mathcal{L}\left(G_{2}\right) C_{n_{2}}=\left(1-\frac{r_{2}}{r_{2}}\right) C_{n_{2}}=0 C_{n_{2}}$. Then

$$
\left(\mathcal{L}\left(G_{2}\right) \bullet B\left(G_{2}\right)\right) C_{n_{2}}=\left(1-\frac{r_{2}}{r_{2}+r_{1}}\right) C_{n_{2}}=\frac{r_{1}}{r_{2}+r_{1}} C_{n_{2}}
$$

and

$$
\left(\lambda I_{n_{2}}-\left(\mathcal{L}\left(G_{2}\right) \bullet B\left(G_{2}\right)\right)\right) C_{n_{2}}=\left(\lambda-\frac{r_{1}}{r_{2}+r_{1}}\right) C_{n_{2}}
$$


Also, $C_{n_{2}}^{T} C_{n_{2}}=\frac{n_{2}}{\left(r_{1}+r_{1} n_{2}\right)\left(r_{2}+r_{1}\right)}$. Hence,

$$
\begin{aligned}
\chi_{G_{2}}\left(B\left(G_{2}\right), C_{n_{2}}, \lambda\right) & =C_{n_{2}}^{T}\left(\lambda I_{n_{2}}-\left(\mathcal{L}\left(G_{2}\right) \bullet B\left(G_{2}\right)\right)\right)^{-1} C_{n_{2}} \\
& =\frac{n_{2}}{\left(r_{1}+r_{1} n_{2}\right)\left(r_{2}+r_{1}\right)\left(\lambda-\frac{r_{1}}{r_{2}+r_{1}}\right)} .
\end{aligned}
$$

Again, since $\mathcal{L}\left(G_{1}\right) \bullet B\left(G_{1}\right)=I_{n_{1}}-\frac{1}{r_{1}+r_{1} n_{2}} A\left(G_{1}\right)$, we get

$$
\begin{aligned}
\mathcal{L}\left(G_{1}\right) \bullet B\left(G_{1}\right) & =\frac{1}{r_{1}+r_{1} n_{2}}\left(r_{1} n_{2} I_{n_{2}}+r_{1} \mathcal{L}\left(G_{1}\right)\right) \\
& =\frac{1}{n_{2}+1}\left(n_{2} I_{n_{2}}+\mathcal{L}\left(G_{1}\right)\right) .
\end{aligned}
$$

Now, if $\delta_{j}$ is an eigenvalue of $\mathcal{L}\left(G_{2}\right)$ and $\mu_{i}$ is an eigenvalue of $\mathcal{L}\left(G_{1}\right)$ then,

$$
\begin{aligned}
f_{G_{1} \star G_{2}}(\lambda)= & \prod_{j=1}^{n_{2}}\left(\lambda-\frac{1+r_{2} \delta_{j}}{r_{2}+1}\right)^{n_{1}} \\
& \times \prod_{j=1}^{n_{2}}\left(\lambda-\frac{n_{2}+\mu_{i}}{n_{2}+1}-\frac{n_{2} r_{1}\left(\mu_{i}^{2}-2 \mu_{i}+1\right)}{\left(n_{2}+1\right)\left(r_{2}+r_{1}\right)\left(\lambda-\frac{r_{1}}{r_{2}+r_{1}}\right)}\right) .
\end{aligned}
$$

(i) Since the only pole of $\chi_{G_{2}}\left(B\left(G_{2}\right), C_{n_{2}}, \lambda\right)=\frac{n_{2}}{\left(r_{1}+r_{1} n_{2}\right)\left(r_{2}+r_{1}\right)\left(\lambda-\frac{r_{1}}{r_{2}+r_{1}}\right)}$ is $\lambda=\frac{r_{1}}{r_{2}+r_{1}}$ and 0 is an eigenvalue of $\mathcal{L}\left(G_{2}\right)$, then $\frac{r_{1}+r_{2} \delta_{j}}{r_{2}+r_{1}}$ is an eigenvalue of $\mathcal{L}\left(G_{1} \star G_{2}\right)$ with multiplicity $n_{1}$ for $j=2, \ldots, n_{2}$.

(ii) The remaining $2 n_{1}$ eigenvalues are obtained by solving the equation

or

$$
\lambda-\frac{n_{2}+\mu_{i}}{n_{2}+1}-\frac{n_{2} r_{1}\left(\mu_{i}^{2}-2 \mu_{i}+1\right)}{\left(n_{2}+1\right)\left(r_{2}+r_{1}\right)\left(\lambda-\frac{r_{1}}{r_{2}+r_{1}}\right)}=0 .
$$

$\left(n_{2}+1\right)\left(r_{2}+r_{1}\right) \lambda^{2}-\left(r_{1}+2 r_{1} n_{2}+r_{2} n_{2}+r_{1} \mu_{i}+r_{2} \mu_{i}\right) \lambda+r_{1} \mu_{i}\left(1-n_{2} \mu_{i}+2 n_{2}\right)=0$.

So the eigenvalues are,

$$
\lambda_{i}=\frac{\left(r_{1}+2 r_{1} n_{2}+r_{2} n_{2}+r_{1} \mu_{i}+r_{2} \mu_{i}\right) \pm \sqrt{\left(r_{1}+2 r_{1} n_{2}+r_{2} n_{2}+r_{1} \mu_{i}+r_{2} \mu_{i}\right)^{2}-4 r_{1} \mu_{i}\left(r_{2}+r_{1}\right)\left(n_{2}+1\right)\left(1-n_{2} \mu_{i}+2 n_{2}\right)}}{2\left(r_{2}+r_{1}\right)\left(n_{2}+1\right)}
$$

for $i=1,2, \ldots, n_{1}$.

Example 2.1. Let us consider $G_{1}=C_{4}$ and $G_{2}=K_{3}$. Then the normalized Laplacian eigenvalues of $G_{1}$ are $1-\cos \frac{2 \Pi k}{4}$ for $k=0,1,2,3$ and the normalized Laplacian eigenvalues of $G_{2}$ are 0 and $\frac{3}{2}$ with multiplicity 2 .

Now using the result of Theorem 2.1, we get the normalized Laplacian spectrum of $G_{1} \circ G_{2}$, as

$$
\left.\left.\left\{\frac{4}{3} \text { (multiplicity } 8\right), 0, \frac{14}{15}, \frac{10 \pm \sqrt{70}}{15} \text { (multiplicity } 2\right), \frac{13 \pm \sqrt{109}}{15}\right\} .
$$


From Theorem 2.2, we get the normalized Laplacian spectrum of $G_{1} \diamond G_{2}$, as

$$
\left\{\frac{5}{4} \text { (multiplicity 8), } 0, \frac{5}{4}, \frac{3 \pm \sqrt{2}}{4} \text { (multiplicity 2), } \frac{7 \pm \sqrt{3}}{8}\right\} \text {. }
$$

Finally by Theorem 2.3, we get the normalized Laplacian spectrum of $G_{1} \star G_{2}$, as

$$
\left\{\frac{5}{4} \text { (multiplicity 8), } 0, \frac{5}{4}, \frac{1}{2} \text { (multiplicity 2), } 1 \text { (multiplicity } 2 \text { ), } \frac{7 \pm \sqrt{33}}{8}\right\} \text {. }
$$

Remark 2.1. If $G_{1}$ and $G_{2}$ are two regular graphs then we find from Theorems 2.1, 2.2 and 2.3, that the normalized Laplacian spectrum of all the coronas depend only on the degrees of regularities, number of vertices, number of edges, and normalized Laplacian eigenvalues of $G_{1}$ and $G_{2}$. Thus for $i=1,2$, if $G_{i}$ and $H_{i}$ are $\mathcal{L}$-cospectral regular graphs then $G_{1} \circ G_{2}$ (resp. $G_{1} \diamond G_{2}$ and $\left.G_{1} \star G_{2}\right)$ is $\mathcal{L}$-cospectral with $H_{1} \circ H_{2}$ (resp. $H_{1} \diamond H_{2}$ and $H_{1} \star H_{2}$ ).

Now we apply the results of the paper and determine some normalized Laplacian cospectral graphs. Since for an $r$-regular graph $G$ we have $\mathcal{L}(G)=I_{n}-\frac{1}{r} A(G)$, the Lemma below is immediate.

Lemma 2.2. Two regular graphs are $\mathcal{L}$-cospectral if and only if they are cospectral.

In the literature there are several regular cospectral graphs, for example see [15]. In Theorem 2.4 below we construct non-regular $\mathcal{L}$-cospectral graphs using coronas. Proof of this theorem follows from Remark 2.1 and Lemma 2.2.

Theorem 2.4. If $G_{1}$ and $H_{1}$ (not necessarily distinct) are $\mathcal{L}$-cospectral regular graphs, and $G_{2}$ and $H_{2}$ (not necessarily distinct) and not necessarily different from $G_{1}$ and $H_{1}$, are $\mathcal{L}$-cospectral regular graphs, then $G_{1} \circ G_{2}$ (resp. $G_{1} \diamond G_{2}$ and $G_{1} \star G_{2}$ ) and $H_{1} \circ H_{2}$ (resp. $H_{1} \diamond H_{2}$ and $H_{1} \star H_{2}$ ) are $\mathcal{L}$-cospectral graphs.

Example 2.2. Applying Theorem 2.4 here we construct $\mathcal{L}$-cospectral graphs. We consider regular cospectral graphs $G_{1}$ and $H_{1}[15]$ as given in Figure 1.
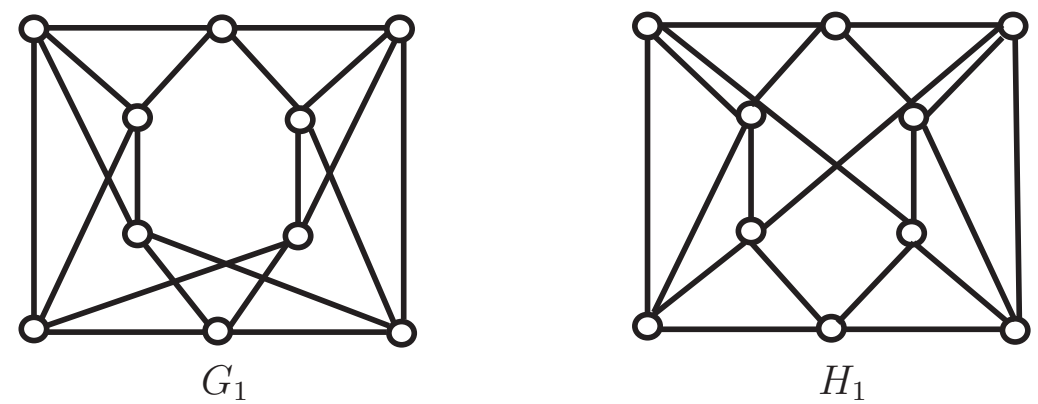

Figure 1. Two cospectral regular graphs.

We also consider graphs $G_{2}$ and $H_{2}$ both of which are copies of $K_{2}$. Now by Theorem 2.4 graphs $G_{1} \circ K_{2}$ and $H_{1} \circ K_{2}$ given in Figure 2a and Figure 2b respectively are 
$\mathcal{L}$-cospectral. Similarly graphs $G_{1} \diamond K_{2}\left(\right.$ resp. $\left.G_{1} \star K_{2}\right)$ and $H_{1} \diamond K_{2}\left(\right.$ resp. $\left.H_{1} \star K_{2}\right)$ are also $\mathcal{L}$-cospectral graphs.

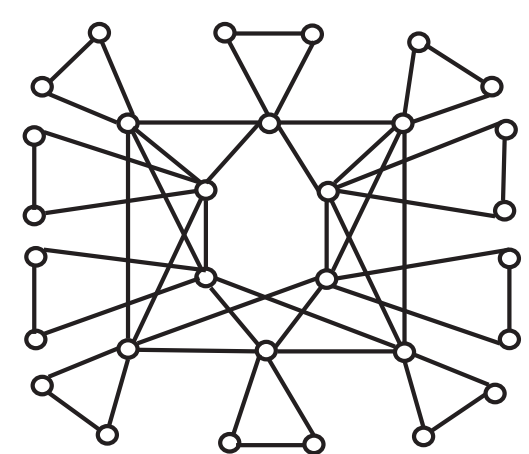

(a)

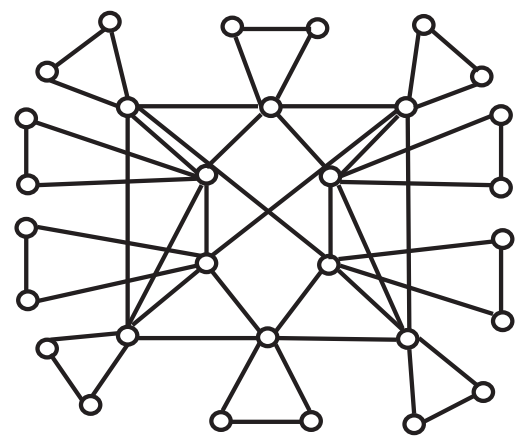

(b)

FIgURE 2. Non-regular nonisomorphic $\mathcal{L}$-cospectral graphs.

\section{REFERENCES}

[1] A. Banerjee and J. Jost, On the spectrum of the normalized graph Laplacian, Linear Alg. Appl. 428 (2008), 3015-3022.

[2] S. Barik, S. Pati and B. K. Sharma, The spectrum of the corona of two graphs, SIAM J. Discrete Math. 24 (2007), 47-56.

[3] S. Butler and J. Grout, A construction of cospectral graphs for the normalized Laplacian, Electron. J. Combin. 18 (2011), Article ID 231.

[4] G. Chen, G. Davis and F. Hall, An interlacing result on normalized Laplacians, SIAM J. on Discrete Math. 18 (2004), 353-361.

[5] F. R. K. Chung, Spectral Graph Theory, CBMS Regional Conference Series in Mathematics, Vol. 92, American Mathematical Society, 1997.

[6] D. Cvetković, P. Rowlinson and S. Simić, An Introduction to the Theory of Graph Spectra, Cambridge University Press, 2009.

[7] I. Gopalapillai, The spectrum of neighborhood corona of graphs, Kragujevac J. Math. 35 (2011), 493-500.

[8] F. Harary, Graph Theory, Addison-Wesley, Reading, PA, 1969.

[9] R. A. Horn and C. R. Johnson, Topics in Matrix Analysis, Cambridge University Press, Cambridge, 1991.

[10] Y. Hou and W.-C. Shiu, The spectrum of the edge corona of two graphs, Electron. J. Linear Algebra 20 (2010), 586-594.

[11] H. H. Li and J. S. Li, A note on the normalized Laplacian spectra, Taiwanese J. Math. 15 (2011), 129-139.

[12] X. Liu and S. Zhou, Spectra of the neighborhood corona of two graphs, Linear Multilinear Algebra 62 (2013), 1205-1219.

[13] C. McLeman and E. McNicholas, Spectra of coronae, Linear Algebra Appl. 435 (2011), 9981007.

[14] S. Wang and B. Zhou, The signless Laplacian spectra of the corona and edge corona of two graphs, Linear Multilinear Algebra 20 (2012), 1-8.

[15] E. R. van Dam and W. H. Haemers, Which graphs are determined by their spectrum?, Linear Algebra Appl. 373 (2003), 241-272. 
${ }^{1}$ Department of Mathematics, Indian Institute of TECHNOLOGy KharagPuR, KHARAGPUR, INDIA-721302

E-mail address: arpita.das1201@gmail.com

E-mail address: pratima@maths.iitkgp.ernet.in 